\title{
Using the Notion of 'Information Flow' to Investigate Why a Signal May Stand Differently for Individuals
}

\author{
Rashmi Mantri ${ }^{1}$, Junkang Feng ${ }^{1,2}$ \\ ${ }^{1}$ Database Research Group, School of Computing, University of the West of Scotland, Scotland, UK; ${ }^{2}$ Business College, Beijing \\ Union University, Beijing, China. \\ E-mail: \{Rashmi.mantri, Junkang.feng\}@uws.ac.uk
}

Received July $23^{\text {rd }}, 2010$; revised August $22^{\text {nd }}, 2010$; accepted October $9^{\text {th }}, 2010$.

\begin{abstract}
Studies over past century show that the organisations profoundly rely on signs (signals in general) and norms to operate. Information systems may be seen as systems of signs. Signs are indispensible part of norms, which people follow in order to act in an organisation. A signal or sign may convey different messages to different agents. If we eliminate merely subjective interpretations, which could be wrong from the receiver's perspective, it must be the case that for such a phenomenon to happen the signal does carry different information for different individuals in the sense of actually informing them. We explore how this is possible in this paper. We observe that information theory and semiotics are strongly related. Therefore, rationally linking organisational semiotics and information theory in some way would be beneficial and highly desirable. Our approach is based upon semantic information and information flow theories put forward by Dretske, Barwise and Seligman along with basic notions of Stamper's organisational semiotics, i.e. sign and norms and Devlin's constraints. We exploit an $S-B-R$ (information source $(S)$ - information bearer $(B)$ - information receiver $(R))$ framework, which incorporate the above mentioned theories to examine information creation, flow and receiving. We put forward the phenomena of information nesting, norms with which a signal or sign is involved and co-existing multiple $S-B-R$ structures to elucidate this fact.
\end{abstract}

Keywords: Information Content, Information Nesting, Semantic Content, Digitalization of Information, Propositional Content

\section{Introduction}

Organisational semiotics [1] is concerned itself with how signs and norms are used for an organisation to operate. A signal (or a sign) carries information. The creation of information is independent of the carrier or receiver, if any, and thus it is objective [2-6] in nature. Individual cognitive agents perceive and interpret the same signal differently, and each interpretation may nevertheless be legitimate. We wonder why this might be the case? If we remove the purely subjective and wrong interpretations from this process, then it must be the case that a signal does in fact carry multiple and different pieces of information and individuals respond to some but not necessarily all of information that is carried.

It seems that information theory and semiotics have a close relation as Hammerstingl [7] says

'It might be said that semiotics belongs under the greater umbrella of communication theory. Some of the work in this field dates back more than half a century such as the work of Shannon and Weaver.'
Organizational semiotics associates two primitive concepts - the sign and norm to understand an organization and their use of different signs, which consequently helps us build a scientific study of information and information systems.

Synthesising and incorporating a range of relevant theories including Stamper and Liu's organisational semiotics [2,8], Dretske, Barwise and Perry, Barwise and Seligman's semantic information theories of information flow [3,5] and Devlin's [9] constraints, under a coherent framework called S-B-R, we explore the following questions:

- May different observers receive different information from the same signal?

- If so, what is the mechanism whereby the above happens?

Two terms are employed, i.e., analogue and digital, to explore this idea further. Carr [10] states that analogue signals are continuous like images and sound, whereas digital signals are discrete in nature. The natural world is analogue $[11,12]$ i.e. there is plenty of information avai- 
lable, but it is not specific and clear, whereas digital information is precise, limited and exact like distinction between true/false, $0 / 1$ etc. This phenomenon is explained by the notion of digitalising analogue information by humans.

By evaluating information content and information nesting we explore how one piece of information is nested in other. Various agents, employing their $3 \mathrm{rd}$ order of intentionality [3], identify one piece of information among enormous available, which then becomes the semantic content of their belief.

The rest of the paper is organised as follows. In Section 2 , we define frequently used terms in this paper, i.e., information, signal and sign. In Section 3, we discuss the S-B-R framework. In Section 4, we look at information content and information nesting. In Section 5, we explore how to receive information through digitalization. Section 6 analyse the roles of constraints and those of norms. Section 7 looks at co-existing multiple S-B-R structures. We give concluding remarks in Section 8.

\section{Information, Signal and Sign}

\subsection{Information}

Information is central to the most contemporary information systems, however, there is no consensus on the nature of information. Various definitions of information are given, some define information as objective while others do subjective, for example:

- Intuitively [6], "information is often used to refer to non-mental, user-independent, declarative, semantic contents which are embedded in physical implementations like databases and encyclopedias and can be produced, collected, accessed and processed."

- According to the Cambridge Dictionary of Philosophy [13], information is: an objective (mind independent entity. It can be generated and carried by messages (words, sentences) or by other products of interpreters. Information can be encoded and transmitted, but the information would exist independently of its encoding or transmission." The information is therefore taken as user independent, objective entity and does not depend on observer.

- According to Shannon and Dretske [3,14] information is generated due to reduction in uncertainty and it must be contingently true (this is discussed in detail in Section 3 and been used as a base of this paper).

- Three quotations from a variety of texts well depict the fame of the bipartite account (having two parts, i.e., information: data + meaning):

- Information is data that has been processed into a form that is meaningful to the recipient. Davis and Olson [15].

- Data is the raw material that is processed and refined to generate information. Silver and Silver [16].
- Information equals data plus meaning. Checkland and Scholes [17].

Even after gaining a sufficient consensus the bipartite account is questionable and needs more accurate formulation. However, we strongly believe that information is a mind independent entity and in the remainder of this paper, we assume such an objective characteristic of information.

We take the following as the basis of our definitions of signal and sign:

\subsection{Signal}

- A signal is something that encodes a message, i.e., information, and conveys it from one place to another Saenz-Ludlow [18].

- Any sign, gesture, token, etc., that serves to communicate information - Farlex Dictionary [19].

- An indicator, such as a gesture or colored light, that serves as a means of communication - Farlex Dictionary [19].

\subsection{Sign}

- When x stands for y or signifies y then it is signified as sign - Stamper [1]. As mentioned earlier semiotics framework $[1,20]$ is a useful tool while studying the nature of information as information exists and carried by signs.

- Something that suggests the presence or existence of a fact, condition, or quality - Farlex Dictionary [19].

So it looks as if signal is more general and non-persistent, and a sign seems fixed by conventions. Signs are special types of signals and we will use the two terms interchangeably in this paper.

\section{S-B-R Framework}

S-B-R stands for information source, information bearer and information receiver. The literature is full of various definitions of information and it still remains an 'explicandum term' according to the Floridi [6]. The Mathematical Model of Communication, given by Shannon and Weaver [2], covers only engineering side of information transmission through a communication channel. In this model probability is used to define amounts of information [2], which is a result of 'reduction in uncertainty'. Instead Dretske [3] focused on the content and therefore the semantic side of information. According to Dretske [3] information is objective and it is being carried, but he did not mention that to carry or bear that information, a 'particular' concrete real world object is necessary. Therefore, he does not seem to clearly distinguish between the information source and information bearer. Devlin [9] on the other hand gives an abstract model in terms of 'infon' and based upon situation theory [4], 
goes on describe how a receiver acquires information. Nevertheless, Devlin does not seem to say much about the relationship between a source of information and the information bearer in question.

Therefore, to incorporate various theories [1-5,9] into a coherent whole, to facilitate further studies of information and information system development, $\mathrm{Hu}$ and Feng [21] identified the aforementioned gap and developed an overarching model, called the S-B-R framework. The key ideas behind developing such a model are as follows:

- To gain insight of, a variety of, phenomena in human communication, to understand information creation and transmission and advancement of an existing information systems.

- To reconcile and integrate a range of relevant theories including organisational semiotics $[1,22]$ and theories about semantic information and information flow $[1-5,9]$. This enables us to pay attention to social layers semantics, pragmatics and social world as well as technical layers - physical world, syntactic layers and empirics.

- To study information and information flow not only concerning 'amounts of information' but also 'contents of information'.

We summarise the S-B-R framework in the paragraphs that follow.

\subsection{Information Source}

Following Shannon and Dretske $[3,14]$ a selection process may be seen as an information source when reduction in uncertainty takes place. Such a process results in a si- tuation, any event or uncertain outcome etc. For instance, 'choosing one of the traffic instructions 'to stop' out of available instructions (Figure 1)' could be an information source S. According to Falkenberg et al. [23] an information source can be seen as 'sign object', from the semiotics perspective. It follows the definition of 'sign' given by Pierce [23] that it is a thing which a sign refers to. Our ontological assumptions are that the creation and existence of information is independent of receivers.

\subsection{Information Bearer}

A traffic light, a sign, a signal, a symbol, or an information system could be seen as an information bearer. For example, a 'red traffic light' carries information that, the instruction to traffic is 'to stop'. According to Stamper [1], if $x$ stands for $y$ then it works as a sign. Here $x$ is an information bearer for $y$. A sign carries information to people and therefore serves as a representation within a representation system [24].

Moreover we consider that the literal meaning of the bearer/carrier (used synonymously in this paper), if any, is independent of the information it bears and it is only a coincidence if the former is part of the latter. Following this we maintain that according to Falkenberg et al. [23], 'representamen', e.g. a red traffic light - thing which is serving as the sign's carrier, is not depending on its meaning. For instance a database's column name might refer to something which has no semantic relation with its meaning of the given name.

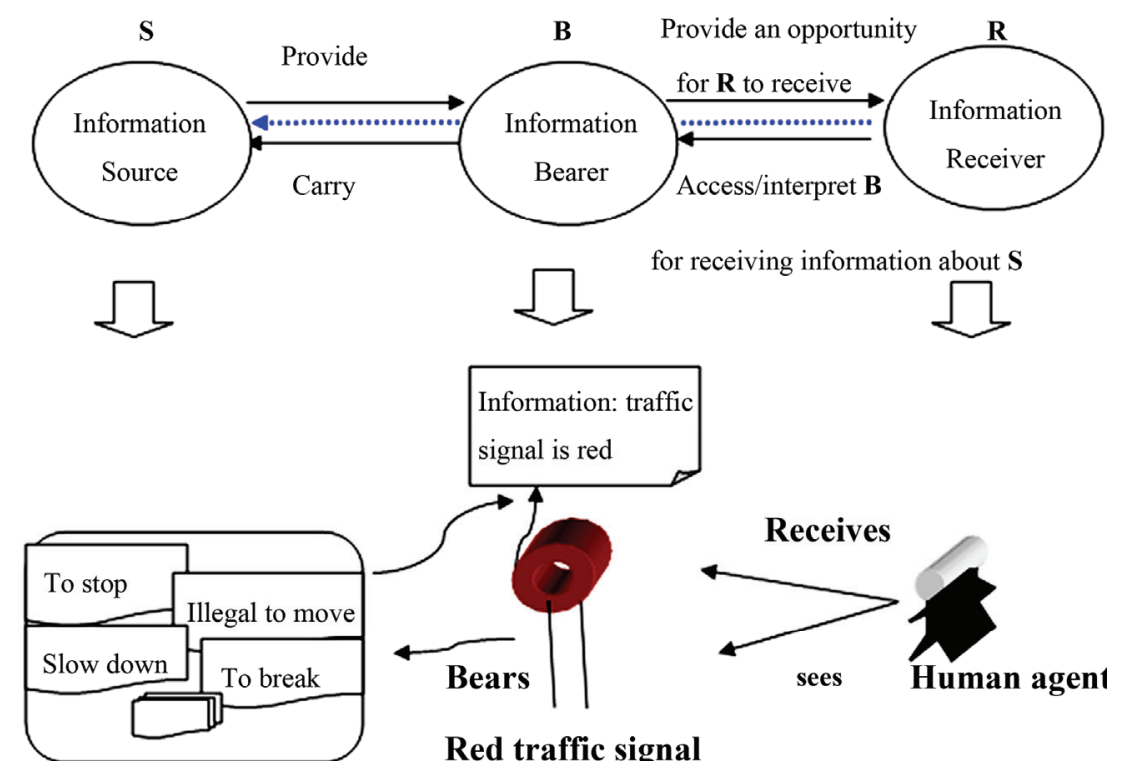

Information source

Information bearer

Information receiver

Figure 1. S-B-R framework with an example. 


\subsection{Information Receiver}

An information receiver has to be aware and attuned of relevant constraints [9] in order to extract a piece of information carried by the information bearer. Different receivers may receive different pieces of information that are all carried by the same bearer.

We illustrate this S-B-R framework with the example of red traffic signal to further elaborate our idea.

As shown in Figure 1 that some information at the information source, namely instructions to the traffic is generated due to 'reduction in uncertainty', i.e., 'to stop', among 'to go forward' or 'to turn right'. A red traffic light carries this information. The red light is capable of carrying such information due to certain relation between a bearer and the source, which may be the result of nomic (by natural law) or analytic (by deductive consequence) dependencies [3].

A cognitive agent (an information receiver), for instance, a motorist gets an opportunity to receive the information about the information source by consulting an information bearer.

This is illustrated by a dotted line in Figure 1. Devlin's [9] notion of constraints comes into the scenario at this juncture, that is, if the human agent as information receiver is familiar with some constraint, namely 'red traffic signal $\rightarrow$ to stop', then he would receive the instruction 'to stop' by seeing the red traffic light. These constraints formulate the dependencies and as a result the informational relationship [3] between the information bearer and the source. However, the driver may also receive information that the break of the vehicle should be applied. Moreover, the passenger in the same vehicle may receive the information that the break will squeak. Where do these pieces of information come from? The answer is that these are also carried by the red light, and more precisely they are nested in the information 'to stop'.

\section{Information Content and Information Nesting}

Different pieces of information may link to one another, and one could be nested in another. It seems reasonable to talk here about signal's informational content as different types of information and a range of information pieces are carried by a signal in its information content.

\section{Definition 1: Information content}

According to Drestke [3]:

"A signal $r$ carries the information that $s$ is $F=$ the conditional probability of $s$ 's being $F$, given $r$ (and $k$ ), is 1 (but, given $k$ alone, less than 1)", $\mathrm{k}$ in the parentheses is normally taken as prior knowledge of a person about s. We believe that $\mathrm{k}$ captures the relativism on how we de- fine what is involved in an information source.

For instance, suppose that the information that ' $\mathrm{r}$ is $\mathrm{G}$ ' is carried by a signal say ' $s$ is $F$ ', and $r$ being $G$ in turn carries the information that ' $\mathrm{u}$ is $\mathrm{H}$ ' then this information that ' $\mathrm{u}$ is $\mathrm{H}$ ' is also carried by the same signal, i.e., ' $\mathrm{s}$ is $F$ '. The notion of the 'information content of data' is presented in details in (Xu et al., 2009).

In general terms, whenever there is the information that $\mathrm{r}$ is $\mathrm{G}$, and $\mathrm{u}$ is $\mathrm{H}$ is a result of any natural law or convention, and then the latter is nested in the former. This can be formalised with probability, namely, if with the condition that $r$ is $G$, the probability for $u$ being $H$ is 1 , otherwise it is not 1 , then whenever ' $r$ is $G$ ' is carried, ' $\mathrm{u}$ is $\mathrm{H}$ ' must be carried too.

\section{Definition 2: Nested Information}

Dretske [3] defines nested information as follows: "The information that $t$ is $G$ is nested in s's being $F=s$ 's being $F$ carries the information that $t$ is $G$ '”.

Therefore, if the information that $\mathrm{s}$ is $\mathrm{F}$ carried by a signal, then all the information nested (analytically or nomically) in s's being $\mathrm{F}$ is also carried.

'Analytically nested' is where the nesting is result of deductive consequence of an entity or the dependency is established mathematically. For example, if we say that it is a table then it also carries the information by definition that it is an article of wood supported by one or more vertical legs and having a flat horizontal surface. 'Nomically nested' is where the nesting is followed by scientific and natural laws. For example, a metal iron expands when heated. Then any signal carrying the information that iron is being heated will also carry the information that iron is expanding.

In terms of our traffic light example information nesting could be illustrated as follows. A traffic light being red carries many pieces of information. The most specific information this signal carries is that the driver of a vehicle should stop at the junction. There could be other information nested in the most specific information, for example, it is illegal not to stop, some other reason to stop, they should not move until the light changes and the road is clear. Such a situation is illustrated in Figure 2.

\section{Receiving Information through Digitalization}

As mentioned earlier, two terms, i.e., analogue and digital are used when we look at information being carried by a signal. Following Dretske [3] information can be carried in either digital or analogue form and the most specific information that is carried by a signal is in digital form. All other information is nested in the most specific information, and is in analogue form. Through a process called digitalization, a piece of information in 


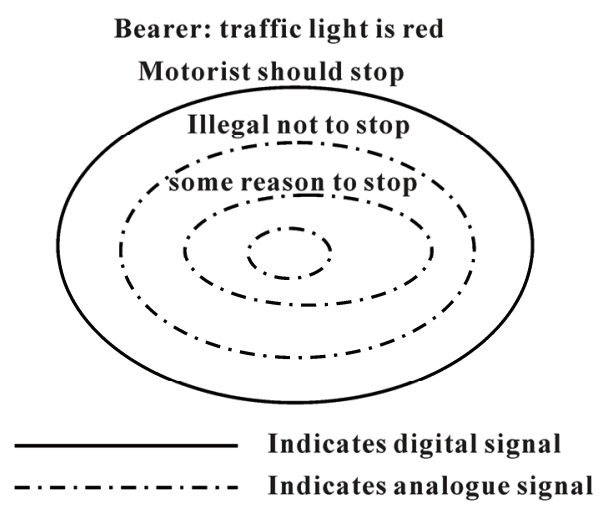

Figure 2. Nested information.

analogue form converts into digital form, i.e., becomes the most specific information, which is normally carried by a cognitive state.

The analogue information that a signal carries is potentially infinite, but bounded. For instance, a picture of a tree is full of analogue information like its colour, shape and location, through digitalization (analogue todigital), we may end up with a statement that it is an Oak. Much information is lost, but the statement is precise, exact and accurate. To receive a particular piece of information, carried by a signal, it is necessary to go through the aforementioned process of digitalization $[3,12,25]$ what is received constitutes part of what is digitalized, that is what the signal meant to the receiver.

We propose that such a course of action is cognitive agent dependent. What gets digitalized becomes the most explicit information for the cognitive agent. A motorist's digitalization of the information carried by the red traffic light is 'to stop'. The passenger does it to get 'the break is going to squeak'. Digitalization is possible only because of the ability of perception and cognition of the cognitive agent.

To explore further how digitalization takes place, we have to appreciate and integrate a few more basic concepts shown below:

\section{Definition 3: Propositional content}

Schiffer [26] defines "To a first approximation, propositional content is whatever that-clauses contribute to what is ascribed in utterances of sentences such as Ralph believes that Tony Curtis is alive or Ralph said that Tony Curtis is alive. An account of propositional content is of foundational importance in the theory of linguistic and mental representation."

The propositional content of a statement is that part which proposes something that may or may not be true. In the examples above the statement may be interpreted as propositional content because it's only a belief or reported statement as opposed to a statement of fact.
Drestke [3] observes that "cognitive states always, either explicitly or implicitly, have a specific propositional content. 'We know or believe, or judge, or think that $s$ is F' by identifying, classifying or categorise s as F."

A propositional statement includes an explicit proposal with which a reader may agree or disagree. However other statements may nevertheless be propositional if they contain implicit assertions.

According to Dretske [3] there are two types of information processing systems: the type which could convert acquired information into knowledge and the other one could not. In this context we talk about the first type of information processing systems that qualify it as of cognitive uniqueness. All information processing systems possess certain low order intentionality referred as first order of intentionality (see below). As discussed earlier, for information bearer to carry the information there is some kind of informational relationship between a source and a bearer.

If a bearer or carrier of an information $\mathrm{S}$, carries the information that $\mathrm{t}$ is $\mathrm{F}$, it does not necessarily carry the information that $t$ is $G$ even though all F's are $G$, which is the opposing the fact of information nesting defined earlier. This is important to understand, that if this is the case, then the informational contents of the carrier is called the propositional content, identified with intentional characteristics. In essence, propositional content are the content of the information which is distinguished or identified by a cognitive agent according to his various cognitive states.

There are various cognitive states like knowing, hoping, wishing, intending or believing, which are involved in the digitalization of information. To meet the requirements for cognitive uniqueness a system must be proficient of revealing higher-order intentional states, i.e., any cognitive states like intending or believing must have higher order of intentionality. As the creatures with the cognition capability could perceive, as a result what we believe, intend or have knowledge about must be distinguishable and noticeable even if their contents are interdependent. To help explain this key point Dretske [3] defines three levels of intentionality.

\section{Definition 4: High orders of intentionality}

1) First order of intentionality (low order)

a. All Fs are $\mathrm{G}$

b. $\mathrm{S}$ has the content that $\mathrm{t}$ is $\mathrm{F}$

c. S does not have the content that $t$ is $G$

When the claim of this triad is consistent, then a state of affairs has a content exhibiting the first order of intentionality. For instance if we say all Herman's children have Diarrhea, if the information that $\mathrm{t}$ is Herman's son is carried by $\mathrm{S}$, then $\mathrm{S}$ 's information content does not 
necessarily carry that $t$ has Diarrhea. This kind of intentionality is exhibited by all information processors.

2) Second order of intentionality (high order)

a. It is a natural law that Fs are $\mathrm{G}$

b. S has the content that $\mathrm{t}$ is $\mathrm{F}$

c. S does not have the content that $t$ is $\mathrm{G}$

When the claim of this triad is consistent, then a state of affairs has a content exhibiting the second order of intentionality. For instance one could know or believe that water is boiling without knowing that the water boils at $100^{\circ} \mathrm{C}$ at sea level, even if there is a natural law which tells us that water boils at $100^{\circ} \mathrm{C}$ at sea level.

3) Third order of intentionality (higher order)

a. It is analytically necessary that Fs be G

b. S has the content that $t$ is $\mathrm{G}$

c. S does not have the content that $t$ is $G$

When the claim of this triad is consistent, then a state of affairs has a content exhibiting the third order of intentionality. For instance one might know that the person was born in 35 A.D. without knowing that he is dead. It is analytically not possible that a person, who was born in 35 A.D. not to be dead, does not make it impossible for one to know that $\mathrm{t}=\mathrm{a}$ person was born 35A.D. without knowing that $\mathrm{t}=$ the person is dead.

A machine does not have higher (i.e., the $2^{\text {nd }}$ and the $3^{\text {rd }}$ ) orders of intentionality with regards to its information content. Humans do. So any propositional content exhibiting the third order of intentionality (identifying an explicit piece of information out of analytically nested information, which is the most imperative to his situation and nothing else) is called the semantic content of a cognitive state.

\section{Definition 5: Semantic content}

According to Dretske [3]

"A structures' semantic contents is that piece of information it carries in 'completely' digitalized form; i.e., structure $\mathrm{s}$ has the fact that $\mathrm{t}$ is $\mathrm{F}$ as its semantic content $=$

a. $\mathrm{s}$ carries the information that $\mathrm{t}$ is $\mathrm{F}$ and

b. s carries no other piece of information, $r$ is $G$, which is such that the information $t$ is $F$ is nested (nomically or analytically) in r's being G".

This definition entails that when $\mathrm{S}$ consists of that $\mathrm{t}$ is $\mathrm{F}$, as its semantic content, then the information that $\mathrm{t}$ is $\mathrm{F}$ is carried by $\mathrm{S}$ is in digital form. But the opposite statement does not hold, i.e., a structure can carry the information in digital form but it does not have this as its semantic content. Semantic content must be of the third order of intentionality, thus no any other information is nested in it as it is the only piece of information for a cognitive state.

The semantic content can be described as a human agent's interpretation of an information-carrying incoming message. This semantic structure actually has some internal interpretation method (depending upon person's objective, context and experience) through which it picks, i.e., digitalizes and highlights one component out of several components of information [22].

Now let us consider the following example. Suppose the incoming signal carries the information that it is a red square. Now depending upon different perceptual phases of the total perceptual-cognitive process this information could be analyzed in a different ways and different semantic structures could be generated. Suppose one interpretation digitalized the information that $t$ is a square and another $t$ is a rectangle. In the latter case the system actually extracted a less specific and possibly more important piece of information. Different internal primary representation of the red square can yield different beliefs about the object depending upon surroundings, purpose, understanding, training, values and intention of the subject.

The realisation of the information reflects the interpreter's ability of understanding and explanation' - Cang and Wang [27]. As a result, from the available analogue information (i.e., information content) a receiver digitalizes (extracts a piece of information from many, which then becomes the semantic content of a cognitive state) only that piece of information which is the most useful for his context, serving his purpose, and more importantly the one he is concerned about.

A person's prior knowledge is also one of the key factors in digitalization of information. For instance, if somebody already knows Scotland's cities like Glasgow and surrounding area well, then saying that UWS is in Paisley makes more sense for him than for one who does not know the area. Similarly this prior knowledge and experience is used by many system engineers and managers to identify the problems of system and an organisation respectively in comparison to any novice employee.

\section{Acquisition of Information: Roles of Constraints and Norms}

Consider our red traffic light example again. A red traffic light is the 'representamen' of a sign on the road. What information does one get from it? Devlin's [9] notion of constraints play a crucial role with regards to receiving information as it formulates the relationship between an information source and a bearer. Different people may relate their act with the red light differently, as they are aware and attentive of different constraints. The constraint that the motorist follows and aware could be: 'red traffic light $\rightarrow$ to stop', while for a pedestrian, the relevant and familiar constraint could be: 'red traffic lights $\rightarrow$ to walk across the road'. From above we may con- 
clude that the information the receiver pulls out from the information bearer with regards to the information source relies upon which constraint is invoked. If we consider the case of computer agent in integrating information, e.g. through an intermediary programme like a wrapper, those constraints could be defined as mapping rules functioning between a source schema and an integrated global schema.

Norms and attitudes are other considerations to think about when looking at digitalization. According to Stamper et al. [28], norms are the set of rules that affect actions, behaviour, and ultimately attitudes of people, as norms are created and defined by members of a community. Norms are there in the community and are responsible for social conduct, behaviour, perception, judgement and beliefs. According to Stamper et al. [1,28], a norm is usually in the form of 'if condition then consequent'. We use some examples to illustrate how norms may affect the scope of the content of the given information source.

According to Dretske's theory, an information source consists of some states of affairs and the sum of the probabilities of these states of affairs is one. Now for instance we have a situation where we have to select one of eight employees to attend a conference. For this, we could have Norm 1: If one employee has to go for a conference then all employees will be considered (one of them should be selected randomly).

We will choose one employee randomly following Norm 1. Every employee has the same probability to be chosen. Once one has been chosen, uncertainty is reduced. The probability for that employee to attend the conference becomes 1 from 8 giving $1 / 8$ and following Shannon [14] we see that a possible $\log _{2} 8=3$ bits of information is created. An email or a letter with the name on it would carry this information.

Suppose that we had Norm 2: if one employee has to go for a conference then only female employees will be considered (one of them should be selected randomly). This is an example of behavioural norm [1]. Keeping in mind that we have a situation where four employees are male and four female, then the scope of our information source is changed due to Norm 2. It consists of four states of affaires in place of eight. In this new situation each employee has a probability of $1 / 4$ to be selected to attend the conference. In this case only 2 bits of information is created and then carried.

Suppose that we had Norm 3: if one employee has to go for a conference then only female and tall employees will be considered (one of them should be selected randomly). Keeping in mind that we have only two females who are tall then our information source consists of only two employees due to Norm 3. Each employee has a probability of $1 / 2$ to be selected to attend the conference and only one bit of information is created.

Above examples illustrate that the scope of information source is changed due to modifications in norms. The scope of an information source is of elementary importance for us to investigate the process of information creation, information transmission and reception and here the norms play an important role. Behavioural norms are the ones which determine the agent's behaviour and act under certain conditions, and this type (behavioural) of norms impact more than others.

In essence this discussion of norms describes that norm's existence particularly in an organisation where they are defined as regulatory system; affect our information receiver as he receives the information from the 'representamen' of a sign too. Following Stamper et al. [28] the condition part leads to what is that piece of information which is required to be obeyed by the normsubject (a group or an individual), and the consequent determines the information creation for others through some medium. Consequently alteration of norms in an existing organisation will definitely manipulate the agent's act and the ability of acquiring information. Whenever new norms are introduced, changes in organisational and social behaviour can be observed.

\section{Utilising the Concept of Co-Existing Multiple S-B-R Structures to Further Analyse the Process of Digitalization}

There is another dimension to look at why and how the same sign or signal informs individuals differently. That is, the same sign or signal may be involved in more than one S-B-R structure, which is co-existing. When applying the S-B-R framework to the traffic light, we see that there is more than one such structure in existence as Figure 3 illustrates.

Figure 3 shows that the same sign, the same ' $\mathrm{B}$ ', i.e., a traffic light happening to be red, may be involved in more than one ' $\mathrm{S}-\mathrm{B}-\mathrm{R}$ ' structure, that is, $\mathrm{S}_{1}-\mathrm{B}-\mathrm{R}_{1}$, $S_{2}-B-R_{2}$ and $S_{3}-B-R_{3}$, and therefore carries information about a different source, $S_{i}$. $S_{1}$ is concerned with instructtions to the traffic. $S_{2}$ concerns the functioning of the traffic light systems. $S_{3}$ has indications to pedestrians. Therefore, the same $\mathrm{B}$ provides different $\mathrm{R}$ with different information, and thus has different meaning for them.

As depicted in above diagram, the red traffic light conveys the instruction of 'to stop' to the motorist (Receiver 1), the information that the traffic lights system is working properly to the engineer (Receiver 2) and 'it is safe to walk across the road' to the pedestrian (Receiver 3). Moreover, different S-B-R structure may be related, which is something to be investigated in the future. 


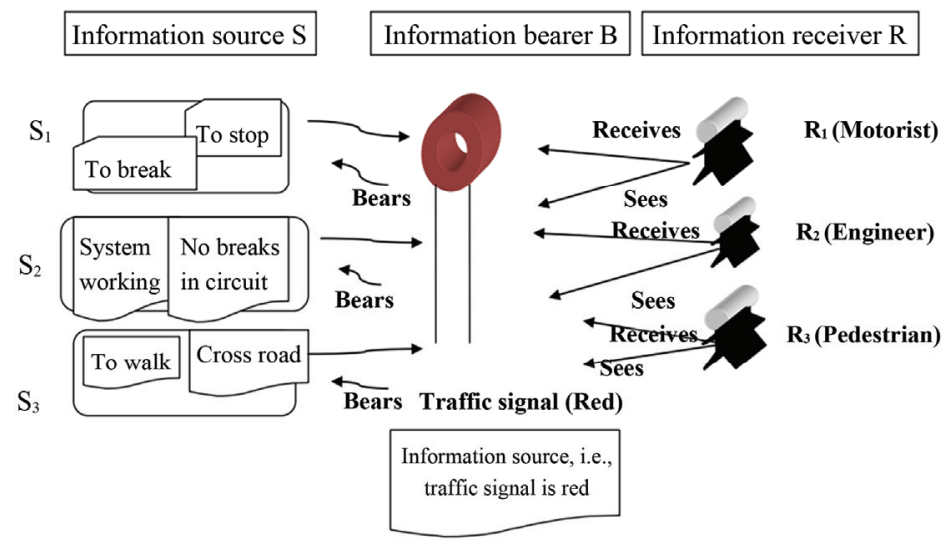

Figure 3. Co-existing multiple S-B-R structures.

\section{Conclusions}

Dretske's notion of information nesting, Stamper's sign, norms and attitudes and Devlin's constraints have been put together under a framework called S-B-R to identify reasons why the same sign may provide different receivers with different information and therefore have different meanings to them. Firstly information is normally nested and the natural world is full of analogue information due to information nesting. A cognitive agent digitalizes available analogue information depending upon his/her cognitive states and a higher order of intentionality exhibited by these cognitive agents. This digitalized information is the most specific information that a cognitive state of the particular agent carries and it is recognised as semantic content of the structure. The second reason is that an information bearer could be involved in more than one S-B-R structure, so different people may choose distinct part of information from different sources and act differently depending upon their intention, interest, prior knowledge, beliefs and values.

\section{Acknowledgements}

This work is partly sponsored by the a grant for Distributed Information Systems Research from the Carnegie Trust for Universities of Scotland in 2007, a grant for research on Semantic Interoperability between Distributed Digital Museums from the Carnegie Trust for Universities of Scotland in 2009 to the corresponding author, and $\mathrm{a} \mathrm{PhD}$ studentship from the University of the West of Scotland, UK to the first author.

\section{REFERENCES}

[1] R. Stamper, "Organizational Semiotics: Information Systems: An Emerging Discipline?" McGraw-Hill, London, 1997.

[2] C. Shannon and W. Weaver, "The Mathematical Theory of Communication," University of Illinois Press, Illinois, 1949.

[3] F. I. Dretske, "Knowledge and the Flow of Information," Cambridge University Press, Cambridge, 1999.

[4] J. Barwise and J. Perry, "Situations and Attitudes," MIT Press, 1983.

[5] J. Barwise and J. Seligman, "Information Flow: The Logic of Distributed Systems," Cambridge University Press, Cambridge, 1997.

[6] L. Floridi, "Is Semantic Information Meaningful Data?" Philosophy and Phenomenological Research, Vol. 70, 2005, pp. 351-370.

[7] W. Hammerstingl, "The Basics of Semiotics." http:// www.olinda.com/edu/CinemaStudies/Semiotics/semiotics. htm

[8] K. Liu, "Semiotics in Information Systems Engineering," Cambridge University Press, Cambridge, 2000.

[9] D. Keith, "Logic and Information," Cambridge University Press, Cambridge, 1995.

[10] J. J. Carr, "Digital Interfacing with an Analogue World," TAB Books, 1979.

[11] A. Wilden, "System \& Structure: Essays in Communication and Exchange," Tavistock, London, 1977.

[12] J. C. Mingers, "Information and Meaning: Foundations for an Intersubjective Account," Information Systems Journal, Vol. 5, 1995, pp. 285-306.

[13] "Cambridge Dictionary of Philosophy," 2nd Edition, Definition of Information, 1999.

[14] C. Shannon, "A Mathematical Theory of Communication," The Bell System Technical Journal, Vol. 27, 1948, pp. 379-423, 623-656.

[15] G. B. Davis and M. H. Olson, "Management Information Systems: Conceptual Foundations, Structure and Development," McGraw-Hill Inc., New York, 1984.

[16] G. A. Silver and M. Silver, "Systems Analysis and Design," Addison Wesley, Massachusetts, 1989.

[17] P. Checkland and J. Scholes, "Soft Systems Methodology in Action," John Wiley \& Sons Ltd (Import), New York, 
1990.

[18] A. Sáenz-Ludlow, "Signs and the Process of Interpretation: Sign as an Object and as a Process," Studies in Philosophy and Education, Vol. 26, No. 3, May 2007, pp. 205-223.

[19] "The Free Dictionary by Farlex. http://www.thefreedictionary.com/

[20] R. Stamper, "Information in Business and Administrative Systems," John Wiley and Sons, New York, 1973.

[21] W. Hu and J. Feng, "Considering Norms and Signs within an Information Source-Bearer-Receiver (S-B-R) Framework," Virtual, Distributed and Flexible Organisations, 2005, pp. 183-184.

[22] K. Liu, R. J. Clarke and P. B. Andersen, "Information, Organisation, and Technology: Studies in Organisational Semiotics," Springer, Berlin, 2001.

[23] D. Falkenberg, W. Hesse, R. K. Stamper, C. Rolland, J. L. H. Oei, P. Lindgreen and B. E. Nilsson, "Framework of Information System Concepts - The FRISCO Report," 1998.
[24] J. Feng and Y. Wang, "NNo Representation without Information Flow': Measuring Efficacy and Efficiency of Representation: An Information Theoretic Approach," $W$. Trans. on Comp., Vol. 8, 2009, pp. 494-505.

[25] J. Mingers, "Cognising Systems: Information and Meaning," Realising Systems Thinking: Knowledge and Action in Management Science, Vol. 25, No. 2, 2006, pp. 103 131.

[26] S. Schiffer, "Propositional Content," Forthcoming in E. Lepore and B. Smith, Eds., Oxford Handbook of Philosophy of Language.

[27] P. Cang and S. Wang, "The Analysis of Uncertain Knowledge Based on Meaning of Information," WSEAS Transactions on Information Science and Application, Vol. 6, 2009, pp. 136-145.

[28] R. Stamper, K. Liu, M. Hafkamp and Y. Ades, "Understanding the Roles of Signs and Norms in Organizations A Semiotic Approach to Information Systems Design," Behaviour \& Information Technology, Vol. 19, 2000, p. 15. 